\title{
Caducidad y vigencia del ensayo en las publicaciones académicas
}

\section{Expiration and validity of the essay in academic publications}

Raimundo Caviedes Hoyos*

\begin{abstract}
Resumen
Tres dispositivos para la educación superior han suscitado las condiciones para el impulso y desarrollo de la investigación científica, la producción académica y las publicaciones, en las Universidades Colombianas, luego de casi un siglo de ser requeridas, por sectores académicos, que abogaban por una educación basada en los principios de la ilustración y la modernidad: La ley 30 de 1992, Los decretos 1444 de 1992 y 1279 del 2002 y finalmente el modelo de medición de grupos de investigación, por parte de Colciencias. La observación, la experiencia y la reflexión crítica sobre el tema permiten afirmar con fundamento, que, paradójicamente, se ha debilitado el ensayo como género crítico de lo actual, y fomentado un pensamiento único de escritura y conocimiento: El artículo científico.
\end{abstract}

Palabras clave: artículo científico, ensayo, investigación científica, pensamiento único, publicaciones, validez.

\begin{abstract}
Three devices for higher education have created the conditions for the imposition and development of scientific research, academic production and publications, in Colombian Universities, after almost a century of being required, by academic sectors, which advocated an education based on the principles of illustration and modernity: Law 30 of 1992, decrees 1444 of 1992 and 1279 of 2002 and finally the model of measurement of research groups, by Colciencias. The observation, the experience and the critical reflection on the subject allow to affirm with foundation, that, paradoxically, the essay has been weakened as a critical genre of the current, and fostered a unique thought of writing and knowledge: The scientific article.
\end{abstract}

Keywords: essay, publications, scientific article, scientific research, unique thinking, Validity.

\section{(c) (1) $\$(0)$}

RECIBIDO: 7 DE JULIO DE 2019 | APROBADO: 8 DE OCTUBRE DE 2019

Cómo CITAR ESTE ARTICULO

Caviedes Hoyos, R. (2020). Caducidad y vigencia del ensayo en las publicaciones académicas. Collectivus, Revista de Ciencias Sociales, 7(1), 25-36. DOI: https://doi.org/10.15648/Collectivus.vol7num1.2020.2552

* Magister en Educación y Filosofía Latinoamericana. Universidad Santo Tomás. Carrera 58 No. 96-106 Apto. 12B Edificio Artefacto Barranqilla. Atlántico, Colombia. raicavi@hotmail.com 


\section{Introducción}

En 1999 fecha en que se publicó “el ensayo", (Caviedes, 1999) escasamente algunas universidades de la ciudad comenzaban a ofrecer programas de posgrados, sobre todo especializaciones y maestrías, y se requería material didáctico de esta naturaleza, que apoyara a los estudiantes en la escritura, uno de cuyos géneros al que se daba mucha importancia, era precisamente el ensayo. Empero fuera de las enciclopedias y diccionarios y uno que otro manual de escritura, se conocía muy poco en la ciudad sobre el tema. Apenas se iniciaba el proceso de modernización de las universidades y aun no eran claros los cambios que ello implicaba para la producción académica de sus profesores, y para la participación de la misma en procesos de competencia, rankings, índice de investigadores, tipos de revistas y de todo lo que tiene que ver con publicaciones en general. Por ello, la finalidad con que se elaboró ese texto de 1999, fue totalmente didáctica.

El I Concurso de Ensayo Cátedra Caribe Orlando Fals Borda 2018, Retorno a la Tierra, fue la oportunidad para reflexionar nuevamente, sobre este interesante género de escritura con una óptica diferente, pensando en su vigencia o decadencia frente al artículo científico, producto más codiciado por las revistas hoy en día, por ser el de mayor categoría para la clasificación en los mejores niveles. Publicar en una revista de alto nivel ( TOP), es el anhelo de muchos investigadores académicos, debido a los cambios ocurridos en el contexto, donde los intereses, las necesidades de las universidades y de los profesores e investigadores, ya son diferentes al simple placer de investigar por la sola satisfacción que produce el descubrir, saber, aportar al conocimiento y solución de problemas de la realidad, sin esperar de esta profusa labor, más que la publicación para dar a conocer a la sociedad en general, y no a un pequeño grupo de expertos, los resultados de la misma.

Conviene recordar que poco antes de la entrada al nuevo milenio eran escasas las universidades en Colombia donde había investigación y donde los medios y recursos para la publicación fueran también, si no abundantes, si, al menos, los necesarios para una buena distribución de la producción académica. Las universidades eran en su mayoría solo centros de enseñanza profesional: no se exigía la investigación; en algunas, donde existían las revistas, los profesores las mantenían muchas veces con recursos propios; la investigación y la escritura tenían un carácter más que todo vocacional; los ascensos al escalafón docente - que solo existían en las universidades públicas- se daban únicamente por la experiencia contabilizada en tiempo, a excepción del último grado, es decir de la titularidad, para la cual se exigía la producción de un texto original de carácter académico, científico o artístico, dependiendo del oficio o la profesión del docente; había solo estímulos a estudios de posgrado pero no eran obligatorios; y, no existía un régimen salarial para los docentes que considerara la producción académica o científica. En casi todos los movimientos estudiantiles universitarios se reclamaba la libertad de cátedra y de investigación, como una reivindicación necesaria para el fomento de la calidad. Ello implicaba destinar, por parte de la institución, un presupuesto para la organización y funcionamiento de la misma.

Las peticiones y reclamos relativos a la investigación por parte de docentes y estudiantes fueron constantes desde el movimiento de reforma educativa de Córdoba de los años 1918- al 30 (Cuneo, 1941), hasta aproximadamente la última década del s. xx, tiempo en el cual, la mayoría de las universidades, especialmente las pequeñas y privadas, que querían pasar de instituciones de educación superior a universidades, comienzan a prestarle más atención a la inversión en investigación. Antes, muchas de estas universidades en Latinoamérica, según De Venanzi (1968) citado en (Tunnermann, 2008) funcionaban con profesores en su mayoría contratados por cátedra, quienes, por sus compromisos por fuera de la universidad, o compartiendo el tiempo con otras universidades, se veían obligados a recitar de memoria 
textos sin aportar nuevos conocimientos que solo son posibles de conseguir, mediante la investigación sea bibliográfica, de laboratorio o de campo.

En los tres momentos considerados como hitos del movimiento estudiantil desde el siglo pasado, puede observarse que siempre ha estado presente como bandera de consigna, la investigación. Ya sea para crearla por inexistente, para impulsarla abogando por mayor presupuesto, o para redireccionarla hacia las necesidades nacionales. Estos momentos han sido:

El movimiento de reforma Universitaria de Córdoba con dimensiones Latinoamericanas ya mencionado. En él, entre otras alusiones a la investigación se lee:

[...]El estudiante tiene la obligación de ser un investigador perenne de la verdad. Sin permitir que el criterio del maestro, ni del libro, sea superior a su razón.

[...] nosotros podemos llevar los claustros, y con mejor derecho que nadie, a los maestros que consideremos aptos para investigar con ellos en los laboratorios del mundo. No es natural que nos sometamos por fuerza a escuchar de un solo profesor y a leer en un solo libro ningún capítulo de la ciencia. Todos estamos dispuestos a estudiar, a trabajar, a darle una interpretación a los problemas sociales, [...]

[...]Que la investigación científica sea severa, pero no ese remedo de investigación hecho por profesionales ad hoc en libros predeterminados. No queremos sustraernos al estudio, sino hacerlo más consciente y más propio, es decir, más intenso y más nuestro. (Cuneo, s.f., pp. 56-64)

El movimiento estudiantil del año 1971, sobre el que puede consultarse entre otros, el Programa mínimo de los estudiantes universitarios de 1971(Acevedo \& González, 2011; Britos, 2016; Hernández, 2007) . Y El movimiento realizado cuarenta años después, en el 2011, dirigido por La Mesa Amplia Nacional Estudiantil (MANE), que también dejó consignado un programa mínimo de reclamaciones, en memoria de su antecesor, el movimiento del 71 (MANE Colombia, 2011)

\section{Estado de la cuestión}

Con los cambios ocurridos en el modelo de universidad y especialmente con la implementación de la investigación y de las publicaciones en las revistas más apetecidas por los investigadores, aquellas que tienen el mayor factor de impacto y que solo reciben artículos científicos (informes de investigación científica) con características y formatos específicos, vale la pena preguntarse si los días del ensayo académico están contados o, si, por el contrario, este sigue vigente. Aunque hoy existe una copiosa bibliografía tanto del ensayo como del artículo científico, hay que señalar, que esta es bastante escasa y marginal, cuando se trata de pensar en los efectos que estos cambios están produciendo en la forma de pensar y de profundizar en el conocimiento de la realidad, y de la manera como los conocimientos se exponen o publican para la comprensión y crítica de la comunidad académica y científica. Aquí se abordará la cuestión bajo la forma del análisis del artículo científico y todo el andamiaje que lo rodea vs. el ensayo.

\section{Método}

Por un lado se ha empleado la observación participativa sobre la forma de implementación de los cambios en los procesos administrativos y organizativos de la investigación en algunas universidades, la participación en la preparación documental para las programaciones de visitas de pares académicos a los diferentes programas con fines de certificación y acreditación, la asistencia a las sesiones de presentación ante pares académicos, la conversación con colegas sobre estos temas, fue el método que permitió la asociación de ideas para ver los nexos entre hechos que a primera vista parecían desarticulados 
y caóticos. Por otro lado, el análisis de textos de producción académica y la reflexión alrededor de la cuestión de fondo, relativa a la penetración de la ideología neoliberal en las universidades, manifiesta en la mercantilización de las mismas y del uso del marketing especialmente en la investigación, condujo a la necesaria selección y organización de estas ideas en un proceso de revisión, de hacer y deshacer, hasta conformar el discurso expositivo, tal como se presenta ahora.

\section{El artículo científico y la universidad actual}

Como se explicaba arriba, antes de 1990 eran pocas las universidades colombianas donde existía una política de investigación, y un presupuesto real dedicado a la misma. Entre las universidades públicas, la que gozaba de mayor tradición en investigación era la Universidad Nacional, vinculada durante la rectoría de Gerardo Molina (1944-1948), según (Jaramillo, 2017), al proceso de “modernización gradualista" en el país, que en la universidad se expresa en la modernización de la organización del saber: adopción de planes de diversificación disciplinar y profesional, al tiempo que se crean formas administrativas para legitimarlas, como las facultades, los institutos y especialmente la estructura de departamentos, considerada en ese entonces como " el núcleo primordial de la enseñanza y la investigación”. García, 2000 citado en (Jaramillo, 2007). Precisamente una de las intenciones de Molina al emprender estos cambios era la de inducir a la investigación, pues de hecho consideraba que, "una universidad que no atiende a ella, deja de serlo para trocarse en una simple escuela de oficios" (ob.Cit., p.12). Sin embargo. a pesar de todos estos, y los subsiguientes esfuerzos:

\footnotetext{
Cabe señalar que, sesenta años después de esta tentativa pionera de integrar la investigación como parte de las funciones esenciales de la universidad, y a pesar de la retórica reciente en el país acerca de la "Universidad de investigación", o de la "universidad del conocimiento", la actividad investigativa, como un rol normalizado, normatizado y legitimado del docente apenas comienza a institucionalizarse en Colombia. (ob.Cit., p.12)
}

Si esto es cierto para la Universidad Nacional y para unas pocas universidades de gran tradición, que siempre han estado a la vanguardia de la actividad científica en Colombia, con mucha mayor razón lo es, para el resto de las universidades comparativamente nuevas, o las regionales donde, salvo ligeras excepciones siempre ha sido espinosa la investigación. Pero durante la última década del siglo XX, tres dispositivos fueron definitivos para la institucionalización de la investigación en Colombia y por ende para la publicación de la producción académica, especialmente de los artículos científicos. Ellos son: La ley 30 de 1992; los decretos 1444 de 1992 y el 1279 de 2002 y el modelo de medición de grupos de investigación.

La Ley 30 (1992), al clasificar las instituciones de educación superior en tres categorías:

- a.) Instituciones técnicas profesionales.

- b.) Instituciones universitarias o escuelas tecnológicas. Y

- c.) Universidades, impulsa la investigación científica especialmente a través de la última categoría, pues las Universidades, de acuerdo con esta ley, son las únicas facultadas para adelantar programas en maestrías y doctorados, además de los programas profesionales y disciplinares.

De esta manera si las Instituciones universitarias deseaban ampliar su oferta educativa y competir en mejores condiciones, debían someterse a un proceso de certificación de calidad de sus programas y demostrar experiencia en investigación científica de alto nivel, en un proceso de acreditación, avalado por el Consejo Nacional de Educación Superior (SESU), para lograr la categoría de Universidad.

El decreto 1444 (1992) primero, y luego el 1279 (2002) que regulan el sistema salarial y prestacional de los docentes de las universidades estatales, son los que, del lado de las Universidades públicas, impulsan 
la investigación y la publicación ya que, después de todo, ellas conservan el reconocimiento de universidad y por tanto, no tendrían necesariamente que someterse al proceso de acreditación para adquirir esta categoría; no obstante lo hacen, para mostrar a la comunidad académica que también están cumpliendo con los requisitos e indicadores de calidad de las otras universidades. La ley 30 en su artículo 19 declara que:

Son universidades las reconocidas actualmente como tales y las instituciones que acrediten su desempeño con criterio de universalidad en las siguientes actividades: La investigación científica o tecnológica; la formación académica en profesiones o disciplinas y la producción, desarrollo y transmisión del conocimiento y de la cultura universal y nacional.

El modelo de medición de grupos de investigación creado por Colciencias, para medir la producción académica de grupos de investigación científica en el país, y específicamente en las universidades ha sido un eficaz instrumento de impulso a la investigación. En efecto, El Departamento administrativo de Ciencia Tecnología e innovación (Colciencias, 2012) realiza convocatorias para la medición de grupos y centros de investigación desde 1991. Este modelo de medición se ha ido ajustando y perfeccionando a medida que se reconoce como investigación nuevos productos y actividades como las de formación y desarrollo tecnológico (empresariales, de innovación, consultorías), productos artísticos entre otros y por la adquisición de la plataforma SCienTI. Pero también en atención a ciertas quejas y reclamos provenientes de universidades o investigadores quienes, ya por vanidad, por amor propio o con justa razón, no entendían cómo algunos grupos nuevos e investigadores inexpertos, podían estar en la misma o superior categoría que otros, que tenían una trayectoria mucho mayor en investigación y un importante reconocimiento de su producción, por parte de la comunidad académica y científica, lo cual les hacía ver con desconfianza la eficacia del modelo.

Los tres dispositivos, no puede negarse, han contribuido de una manera importante en los últimos años, a la modernización tecnológica de las universidades, de la investigación y la publicación. En pocos años Colombia ha pasado de una incipiente investigación científica a tener 5.207 grupos reconocidos por Colciencias, en el año 2017, (Colciencias, 2019) y, en solo 2 años, de 2015 a 2017 se pasó de 10.050 investigadores reconocidos entre todas las categorías (Emérito, Senior, asociado, y junior) a 13.001. En el mismo lapso contabilizando solo lo escrito, es decir, desde el artículo de investigación hasta el capítulo de libro, (no se registra la producción artística y arquitectónica, ni las patentes) se brincó de 75.114 a 151.335 productos de nuevo conocimiento (ob. Cit). Es decir, se duplicó la producción textual en solo dos años, cosa que difícilmente ocurre en una fábrica de objetos. En otras palabras, La producción intelectual ha pasado de ser artesanal (Mills, 2003) a una moderna fábrica en serie, de revistas, libros, artículos, patentes e incluso investigadores.

Y no solo por el uso de los potentes medios tecnológicos de que se dispone hoy como el computador, el internet, los softwares de investigación cuantitativa y cualitativa, los de tomar notas como el Evernote, o el hipertexto, entre otros, que desde cualquier sitio permiten acceder a repositorios, bibliotecas, bases de datos, reduciendo notablemente el trabajo de recopilación de información. Sino también por su mal uso y por su abuso. Hoy día a excepción quizás del trabajo de campo o del laboratorio, según sea el caso, sale todo de internet: La bibliografía, los datos, las imágenes, las ideas, las interpretaciones, las críticas cuando las hay, iTodo!

Por otro lado, los dispositivos mencionados y en particular el modelo de medición, son también perversos y hostiles a la calidad de la investigación y de las publicaciones. Han contribuido a la creación de un ambiente tenso donde se privilegia la publicación sobre todo lo demás, i"publica o muere”! es su lema; no interesa mucho si lo que se publica representa algún aporte al conocimiento, a la disciplina o al método; si se dice algo nuevo, si se cuestiona un punto de vista, si se llena un vacío, si se plantea una nueva forma 
de abordar un tema o una interpretación distinta, mientras cumpla con "las instrucciones para los autores" de tal o cual revista. De la misma manera sucede con las citaciones. No importa qué o por qué se cita: si para refutar una tesis o para apoyarla; para ayudar al compañero a mejorar su índice $\mathrm{H}$ o para cumplir con las normas de la revista; o para facilitar la búsqueda de la fuente, en fin, lo importante es ser citado, o citar para ser citado por contraprestación.

De igual forma, se han desencadenado malas prácticas investigativas y aumentado otras, como puede leerse en (Caviedes, 2014; Caviedes, et al., 2014; Muñoz, 2009; Richter-Boix, 2018; Serrano, 2015). Entre éstas se destacan el plagio y la exagerada citación o uso permanente del argumento por autoridad o Ad verecundiam, que la lógica incluye en las falacias. Respecto del plagio, se conoce su parte más visible que es la copia textual, pero muchas veces se pasa por alto el plagio disimulado. Este consiste en un mal parafraseo. Toman la parte a citar sea un párrafo o una frase y cambian algunas palabras por sinónimos y ya está. Con eso pasan incólume, la prueba del antiplagio. Algunos hacen la cita contextual o indirecta, otros no. La exagerada citación consiste en apoyar en un autor cada tesis, cada frase, cada aseveración o negación que se haga. En consecuencia, ya no se lee ni se piensa, sino que se cita, se promueve con esto la pereza mental. En estos textos es muy difícil determinar cuáles son las ideas originales del autor, cuando las hay. Definitivamente este tipo de formatización y estandarización de la ciencia contrariamente a lo esperado mutila el pensamiento.

Las prácticas del archivo personal elaborado meticulosamente con fichas de libros, apuntes personales, ideas, conversaciones, observaciones, notas al margen o acotaciones, ejemplos y subrayados, entre otros, para luego procesarlos y complementarlos con las ideas originales que van surgiendo del reordenamiento del mismo, a partir de las relaciones antes no reconocidas, el investigador industrial las ha cambiado, por el exceso de cita, el copia y pega, el uso de formatos y hormas donde se pierde el estilo propio, la elaboración de productos modulares (Muñós, 2009) cuyas piezas o fragmentos pueden ir de un congreso a otro, de un seminario a otro, de una revista a otra, con solo retocarlos o disponerlos de otro modo. Recuerdan el Palacio de la Risa del que habla (Toffer, 1973), refiriéndose a muebles o aparatos modulares que pueden adecuarse a cualquier situación, como:

[...] el plan fantástico de «Palacio de la Risa», conocido también por «primer gran espacio móvil del mundo». Este plan no requiere un edificio apto para muchos fines, sino lo que es, en realidad, un Mecano de tamaño más que natural, una colección de partes modulares que pueden ser combinadas en una variedad casi infinita de conjuntos... [se] sitúan los módulos en posición y...[se] juntan en la forma temporal que se desea. (p.43)

El Currículum Vitae de Latinoamérica y el Caribe. -CVLAC,- instrumento de recepción electrónico de información individual de la experiencia investigativa y formativa de investigadores, junto con el modelo de medición de grupos - GrupLAC-, ideado en un principio solo con el fin de apoyar y financiar la investigación, y la introducción de la plataforma ScienTI- Colombia, en el año 2001, se han convertido en sofisticados aparatos que recogen información de toda la actividad investigativa, formativa y docente de cada uno de los investigadores y grupos de investigación que participan en las convocatorias. Clasifica los grupos en distintas categorías; pondera las actividades y la producción investigativa a través de la utilización de indicadores, índices, umbrales, entre otros; agrupa o encasilla a los investigadores de acuerdo a su producción científica para lo cual se vale del índice $\mathrm{H}$, indicador que permite hacer un balance entre el número de publicaciones y las citas que recibe de otros investigadores en un tiempo de dos años. Esto ha hecho de estas herramientas una ayuda inestimable para la evaluación, por parte de las universidades hacia los investigadores y de los pares académicos para los procesos de certificación y acreditación universitaria.

Como consecuencia de la clasificación de grupos e investigadores, que obviamente es una manera de evaluar, se ha creado tanto en las universidades como en los investigadores la necesidad de publicar, 
preferiblemente en las revistas que mayor puntaje otorgue el modelo de medición. Es decir, las de mayor impacto a través de las cuales se obtiene el prestigio y el ascenso en las categorías, o sea las que están inscritas en Scopus, que es la base de datos más prestigiosa en todas las ciencias y disciplinas científicas, la cual ofrece además información sobre el número de citas que ha recibido un autor en cada uno de los artículos publicados. Ahora, como todos los investigadores que apliquen a las convocatorias de Colciencias, quieren publicar en estas revistas, también todas las revistas quieren afiliarse a esta base de datos, que las acepta previo un proceso de evaluación, para verificar el cumplimiento de los requisitos, entre los cuales vale mencionar, la revisión de los artículos por parte de pares, o arbitraje académico doble ciego. La estructura organizativa de los artículos es común a todas las revistas independientemente de la disciplina, para posibilitar la consulta, salvo ciertos requisitos o normas para la publicación, que son facultativos de cada revista. Por ejemplo, si utiliza normas APA o VANCOUVER, el tipo o número de letra, número de páginas entre otras. En todo caso, la mayoría de las revistas aceptan solo los siguientes tipos de escritos o textos con sus consiguientes estándares internacionales:

$\gg$ Artículo de investigación científica y tecnológica.

$\gg$ Artículo de reflexión.

$\gg$ Artículo de revisión.

$\gg$ Reseñas.

Las normas admitidas hoy por las publicaciones científicas son las siguientes: la Asociación Americana de Psicología (APA) por sus siglas en inglés, el Instituto de Ingenieros Eléctricos y Electrónicos (IEEE), Moderno Lenguaje de Asociación (MLA) por sus siglas en inglés, las revistas médicas que usan las Normas de Vancouver y el Instituto Colombiano de Normas Técnicas (ICONTEC). Este último hoy en desuso. En todas ellas la estructura de un artículo científico es el mismo con ligeras variaciones: Título, autoría y afiliación, resumen y palabras clave, introducción, materiales y métodos, resultados, discusión, conclusiones, a veces reconocimientos y por último referencias bibliográficas. Con las bases de datos electrónicas existentes, no cabe la menor duda de que en este momento son imprescindibles los resúmenes y las palabras clave en toda publicación, para facilitar la búsqueda y selección de lo pertinente por internet, sin ellos sería imposible una consulta eficiente; los reconocimientos siempre han sido opcionales, pero recomendables por razones éticas; la bibliografía igualmente lo ha sido por razones éticas y de derechos de autor. El resto, el famoso formato: Introducción, Métodos, Resultados y Discusión IMRYD es el que identifica actualmente la organización del artículo científico, pero también lo que moldea y uniforma el estilo, lo que lo convierte en único. Una sola manera de escribir que busca una sola manera de pensar, en su (Orwell \& s.f, 1984) lo expresa del siguiente modo:

\footnotetext{
La intención de la neolengua no era solamente proveer un medio de expresión a la cosmovisión y hábitos mentales propios de los devotos del Ingsoc, sino también imposibilitar otras formas de pensamiento. Lo que se pretendía era que una vez la neolengua fuera adoptada de una vez por todas y la vieja lengua olvidada, cualquier pensamiento herético, es decir, un pensamiento divergente de los principios del Ingsoc, fuera literalmente impensable, o por lo menos en tanto que el pensamiento depende de las palabras. (p.145)
}

El formato IMRYD y los cánones de escritura de artículos ya comentadas normatizan todo: desde cuántas y cuáles deben ser las palabras que de acuerdo a la temática tratada deben ir en cada uno de los apartados, hasta las formas de redactar las citas y de colocar la bibliografía al final, pasando por el estilo de redacción y el contenido que debe llevar cada ítem; ahora la forma solo es impersonal, puesto que la otra manera de redacción, la personal, que le costó el doctorado a Molano, (2014) rayaría con la "subjetividad" ; el estilo, por el que se podía reconocer la obra de un escritor, se pierde; la nota de pie de página, considerada antes, especialmente por los alemanes, De Quincey (2004), no solo como un signo de sabiduría o erudición, sino también como un recurso para estimular en el lector la curiosidad, ahora es tenida como estorbo 
y reducida al mínimo. El método utilizado debe ser el "científico", lo que hace que las ciencias sociales cuando no optan por el paradigma empírico analítico deban seguir luchando por el reconocimiento de su estatus como ciencia, especialmente por el uso de otros métodos a veces más adecuados a sus fines, o por la afirmación de otros tipos de conocimiento diferentes a la ciencia occidental, como lo está haciendo la epistemología del sur o el pensamiento complejo.

Las publicaciones científicas, siguen ceñidas aún al solo reconocimiento de "dos culturas"; por un lado "la ciencia", caracterizada por el uso del método científico que permite la comprobación a través de datos cuantificables y medibles, o "ciencias duras;" y, por otro, la literatura. Las ciencias sociales solo son aceptadas, si acaso, como ciencias blandas, literatura gris, que a no ser que esté apoyada en la medida, las escalas de medición y la estadística en general, debe vérselas, o sortear múltiples dificultades, para publicar en ellas. Por ello, escritos como los de Molano (2014) cuyo método, "Vaya, mire y me cuenta", según dijo en su discurso al recibir el título de doctor Honoris causa, con dificultad podrían seleccionarse para ser publicados en revistas catalogadas en Scopus, no obstante, su valor para el conocimiento de algunos fenómenos sociales en Colombia y en especial de la población campesina.

\section{5. ¿Y el ensayo?}

¿Qué está pasando con el ensayo que había sido el género de escritura más extendido hasta mediados de siglo XX? ¿Ya no está contemplado como otra opción a publicar en las revistas indexadas? ¿debe este adecuar su estructura flexible, a la del artículo científico, sometiéndose también a los moldes y estándares de las revistas, con el consiguiente sacrificio de su naturaleza para ajustarse a los marcos y medidas de las revistas indexadas? ¿es el libro su último refugio?

Sin entrar a definir el término ensayo debido a su ambigüedad y al uso que se le ha dado en todos los campos del saber: Las humanidades, la literatura, las disciplinas sociales, las ciencias naturales e incluso las ingenierías (Cataño, 1992) es preciso enunciar ciertas características que lo distinguen del artículo científico para entender cómo las revistas indexadas actuales, no son el medio más apropiado para el ensayo. Empero, antes de responder las preguntas formuladas arriba es necesario aclarar que el ensayista no está impedido de investigar con tanta rigurosidad, profundidad, habilidad y maestría, como lo puede hacer el investigador científico; tampoco debe ser más imaginativo, curioso, intuitivo, observador y creativo que el "científico", ni menos racional que este último. Es decir, que los esfuerzos del ensayista por llegar a los resultados que se propone, o su mayor o menor aproximación a la "verdad", su verdad como suele decirse hoy, contrariamente a lo que piensan algunos, no son, valorativamente hablando, ni más ni menos, que los de sus compañeros de viaje, los escritores de artículos científicos.

Algunos, como García Nosa, citado en Jaramillo (2007) y Jaramillo (2017), han pensado que la institucionalización del saber en disciplinas, en las universidades y particularmente en la Universidad Nacional, posibilitó el uso del "método científico" en la investigación de la realidad, procurándole mayor rigurosidad y fiabilidad. Ello, según los autores antes mencionados permitió la superación del ensayismo, que hasta mediados del s.xx había sido la forma favorita de difusión del conocimiento y específicamente del pensamiento social y sociológico en Colombia.

En este sentido Cataño (1992) señala que:

[...] cuando a mediados del siglo comenzó la asimilación de la sociología científica en América Latina, sus figuras más representativas -José Medina Echavarría, Gino Germani y Florestán Fernandez-, emprendieron una tarea de refinamiento teórico y de rigor metodológico que contrastó con la espontaneidad conceptual y la indigencia empírica del ensayismo. (p.38) 
Pero para este último sociólogo, la superación del ensayo no está solo en el uso del método científico, sino en la construcción de sistemas teóricos cerrados. Por eso para él, nunca hubo una superación del ensayo como tal, sino solo una diferencia de tono; aquel desbocado y ligado a las letras y las humanidades; este, el ensayo científico, "nutrido de conceptos, teorías y de datos, que contrastan la argumentación y limitan las conclusiones a lo conocido" (p.39). Refiriéndose a la sociología de la posguerra, especialmente la conocida como sociología del desarrollo y de la dependencia, e incluyendo en ellas las investigaciones de Camilo Torres y a Fals Borda, señala que, "no obstante los desarrollos de la sociología latinoamericana durante los últimos cuarenta años, todavía no posee el aliento necesario para la construcción de sistema" (Cataño, 1992) Son escritos a medio camino, entre una "exposición controlada y [otra] suelta" (ob. Cit., p.39). Pero precisamente a lo que el ensayo, como género de la modernidad se opone, es a la pretensión de los grandes sistemas cerrados que tienen la solución final a todas las respuestas que plantea la naturaleza y la sociedad, sean los sistemas filosóficos premodernos o los sistemas científicos positivistas contemporáneos, quienes lo acusan de erudición y falto de rigor.

Compare el lector los dos párrafos que siguen sobre el “amor romántico" y saque sus propias conclusiones. El primero tomado de un ensayo y el segundo como debería redactarse en un artículo científico:

Primer texto:

Después de todo, la definición romántica del amor - «hasta que la muerte nos separe»- está decididamente pasada de moda, ya que ha trascendido su fecha de vencimiento debido a la reestructuración radical de las estructuras de parentesco de las que dependía y de las cuales extraía su vigor e importancia. Pero la desaparición de esa idea implica, inevitablemente, la simplificación de las pruebas que esa experiencia debe superar para ser considerada como «amor». (Bauman, 2003, p9)

Segundo texto;

De un número de 100 parejas consultadas en Barranquilla entre los 25 y cuarenta años, el $10 \%$ manifestó que la persona con la cual vivía era su tercera pareja; el $70 \%$, la segunda y solo un $20 \%$ no habían tenido otra pareja antes. Esto significa, que, en esta ciudad, la expresión "hasta que la muerte nos separe", se convirtió en un enunciado vacío, un simple protocolo que se usa en el rito del matrimonio para formalizar la unión.

Seguidamente o en pie de página debe llevar la siguiente nota: Encuesta realizada en el marco de la investigación sobre las separaciones en Barranquilla. Ver al final la ficha técnica.

El primer párrafo sería, según el cientificismo, un pensamiento retórico, subjetivo, una generalización vaga, sin ningún sustento empírico y por supuesto falto de rigor. Mientras el segundo sería un texto preciso, riguroso, delimitado, con sustento empírico y por tanto verificable.

El caso es que el ensayo, que tanto ha aportado al conocimiento de la realidad y a su transformación, (por ejemplo, el caso del "Manifiesto Comunista") debido a su estructura abierta: Introducción, cuerpo y conclusión, no sometida a regla alguna, salvo las de la buena escritura y el rigor argumentativo cuando es preciso, no encuentra cabida en los formatos de las nuevas revistas indexadas. Se lo impiden, por un lado, sus características de: Flexibilidad, estilo agradable, ameno, a veces agudo, irónico y mordaz, otras divertido y en contadas ocasiones, también aburrido; y, por otro lado, el uso frecuente como medio de convencimiento del ejemplo, el símil, la metáfora, la narración y el relato, pocas veces aceptados por la ciencia por carecer de sustento empírico.

Queda entonces el libro, como opción a los investigadores académicos de las ciencias sociales que quieran publicar ensayos. Sin embargo, este, el libro, para recibir el aval tanto de la institución como de los evaluadores externos, y ser reconocido como producto científico por parte del modelo de medición 
de Colciencias, tiene que ser resultado parcial o total de una investigación científica. Siendo así, los parámetros de medición pasan a ser los mismos exigidos por las revistas. De ahí la tendencia de los nuevos libros a agrupar artículos diversos de varios investigadores, al modo de las revistas, cerrándose de esta manera, otra puerta a los ensayos.

La gran paradoja, es que mientras los académicos e investigadores de nuestras universidades se preocupan cada vez más por la publicación de artículos en las revistas indexadas, los conceptos y teorías básicas, para sustentar teóricamente sus trabajos, los toman generalmente de pensadores, la mayoría ensayistas, que escriben fundamentalmente en libros. Sin que ello signifique que el ensayismo sea su único género, los siguientes autores entre otros, como bien lo señalan (Maric M, Aliaga \& Uribe, 2018) son de obligada consulta para un marco referencial sobre los temas de imaginarios y representaciones: "Emile Durkheim, Cornelius Castoriadis, Gilbert Durand, Michel Maffesoli, Manuel Antonio Baeza, Enrique Carretero, Armando Silva, Serge Moscovicci, Denisse Hodelet, Stuart Hall" (p.9). Para el tema de vida cotidiana, entre otros: Michel De Certau, Norbert Elías, Anthony Giddens, Irving Goffman, Henry Lefebvre y Mauro Wolf. Así mismo se podrían identificar autores ensayistas, para múltiples temas de las ciencias sociales.

\section{Conclusión}

En conclusión, esto último nos proporciona certeza de la necesidad de la conservación del ensayo, pese a la arremetida del cientificismo comercial manifiesto en la modernización de las publicaciones y en la comercialización del saber a través de las mismas. Para ello se debe ofrecer a los investigadores otras opciones de publicación de acceso libre, a las que se les otorgue reconocimiento equivalente al que actualmente se les da a las revistas científicas indexadas.

\section{Referencias bibliográficas}

Acevedo, A., \& González, D. (2011). Movilización y protesta estudiantil en Colombia. Una lectura desde la organización gremial por el cogobierno universitario y la memoria de protagonistas y testigos. Anuario de Historia Regional y de Las Fronteras, 16(1), 221-242. Retrieved from https://www.redalyc.org/ pdf/4075/407539690009.pdf

Bauman, Z. (2003). Amor Líquido. Acerca de la fragilidad de los vínculos humanos. Mexico: F.C.E.

Cataño, G. (n.d.). La artesania intelectual (pág. 25-41). Bogota, Colombia: Universidad Nacional de Colombia .

Cataño, G. (1992). El ensayo sociológico ¿Entre la ciencia y la literatura? En G.

Caviedes Hoyos, R. (1999). El ensayo. Equidistancia, 1, 108-121.

Caviedes Hoyos, R. (2014). La simulación:Una forma de violencia simbólica. Barranquilla: Editorial Universidad Simón Bolívar.

Caviedes Hoyos, R., Silva Silva, A., Garcia Acuña, Y., Fontalvo Peralta, R., \& Orozco Idárraga, A. (2014). Violencias, nuevas subjetividades y política de civilizacion (R. Caviedes Hoyos, Ed.). Barranquilla: Universidad Simón Bolívar. 
Colciencias. (2012). Modelo de Medición de grupos. Retrieved July 18, 2020, from Minciencias website: https:// minciencias.gov.co/sistemas-informacion/modelo-medicion-grupos

Colciencias. (2019). Modelo medición de grupos. Retrieved from Minciencias website: https://minciencias. gov.co/sistemas-informacion/modelo-medicion-grupos

Cuneo, D. (1941). La reforma Universitaria (1918-1930). Caracas: Biblioteca Ayacucho.

De Quincey, T. (2004). Los últimos días de Emanuel Kant . Madrid: Valdemar.

Decreto 1279. (19 de Junio de 2002). Por el cual se establece el régimen salarial y prestacional de lo Docentes de las Universidades Estatales. Bogotá, Colombia : Ministerio de Educación Nacional . Recuperado el 27 de 03 de 2019, de https://www.mineducacion.gov.co/1621/article-86434.html

Decreto $N^{0}$ 1444. (3 de Septiembre de 1922). Por el cual se dictan disposiciones en materia salarial y prestacional para los empleados públicos docentes de las uiversidades públicas del orden nacional. (D. Oficial, Ed.) Bogotá, Colombia. Recuperado el 27 de 03 de 2019, de https://www.mineducacion.gov.co/1621/ articles-103567_archivo_pdf.pdf

Hernández Arteaga, I. (2007). El programa mínimo de los estudiantes colombianos. movimiento estudiantil universitario de 1971 por la universidad. Todo un país. Revista Historia de La Educación Colombiana, 10(10), 29-57.

Jaramillo, J. (2007). Universidad política y cultura: La rectoría de Gerardo Molina en la Universidad Nacional (1944-1948). Bogota, Colombia: Quiron Revista de estudiantes de Historia.

Jaramillo, J. (2017). Estudiar y hacer sociología en Colombia . Bogota, Colombia: Universidad Central.

Ley 30. (28 de dic de 1992). Congreso de Colombia. Recuperado el 16 de 03 de 2019, de https://www.cna.gov. co/1741/articles-186370_ley_3092.pdf

MANE Colombia. (2011). Programa Mínimo del Movimiento Estudiantil Universitario Colombiano. Retrieved July 18, 2020, from Viva la Mane website: http://manecolombia.blogspot.com/2011/10/programa-minimodel-movimiento.html

Maric M, Aliaga, F., \& Uribe, C. (2018). Estado del arte en imaginarios y representaciones :Un mapa necesario para buscarcaminos investigativos en Iberoamérica. En F. Aliaga, \& M. e. Maric, Imaginarios y representaciones sociales. Estado de la investigación en Iberoamérica (págs. 9-20). Bogota, Colombia: USTA.

Mills, C. W. (2003). La Imaginación sociológica. México: FCE.

Molano, A. (2014). Vaya, mire y me cuenta. Retrieved from En el puente: A las seis es la cita website: https:// yezidarteta.wordpress.com/2014/09/25/vaya-mire-y-me-cuenta/

Muñoz, S. (2009). Los devaneos del docto. El caso de la teoría de consumo cultural en América Latina. Cali: Archivos del Îndice .

Orwell, G., \& s.f. (1984). 1984. In Escuela de Filosofía Universidad ARCIS. Retrieved from http://philosophia. cl/biblioteca/orwell/1984.pdf 
Richter-Boix, A. (2018, June 10). Publica o muere: el lema que promueve la mala ciencia. La Mala Ciencia. Retrieved from https://andaresdelaciencia.com/2018/06/10/publica-o-muere-el-lema-que-promueve-lamala-ciencia/

Serrano, Y. (2015). El Estado en Cuba. Reflexiones en torno a su encargo social en relación con las familias. Collectivus, Revista de Ciencias Sociales, 2(2), 74-100. https://doi.org/10.15648/coll.2.2015.5

Toffer, A. (1973). El Shock del futuro. Barcelona: Plaza \& Janés .

Tunnermann, C. (2008). Noventa años de reforma Universitaria de Córdoba (1918-2008). Buenos Aires: CLACSO. 